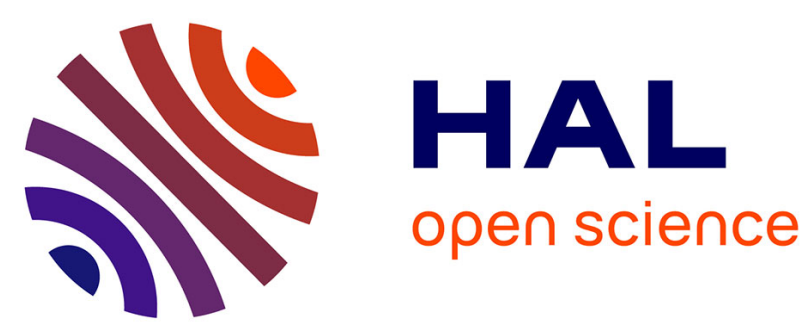

\title{
Blind Source Separation in Nonlinear Mixture for Colored Sources Using Signal Derivatives
}

\author{
Bahram Ehsandoust, Masoud Babaie-Zadeh, Christian Jutten
}

\section{To cite this version:}

Bahram Ehsandoust, Masoud Babaie-Zadeh, Christian Jutten. Blind Source Separation in Nonlinear Mixture for Colored Sources Using Signal Derivatives. LVA/ICA 2015 - 12th International Conference on Latent Variable Analysis and Signal Separation, Technical University of Libere, Aug 2015, Liberec, Czech Republic. pp 193-200, 10.1007/978-3-319-22482-4_22 . hal-01226466

\section{HAL Id: hal-01226466 https://hal.science/hal-01226466}

Submitted on 9 Nov 2015

HAL is a multi-disciplinary open access archive for the deposit and dissemination of scientific research documents, whether they are published or not. The documents may come from teaching and research institutions in France or abroad, or from public or private research centers.
L'archive ouverte pluridisciplinaire HAL, est destinée au dépôt et à la diffusion de documents scientifiques de niveau recherche, publiés ou non, émanant des établissements d'enseignement et de recherche français ou étrangers, des laboratoires publics ou privés. 


\title{
Blind Source Separation in Nonlinear Mixture for Colored Sources Using Signal Derivatives
}

\author{
Bahram Ehsandoust ${ }^{1,2}$, Masoud Babaie-Zadeh ${ }^{2}$, and Christian Jutten ${ }^{1}$ \\ 1 Univ. Grenoble Alpes, CNRS, GIPSA-lab, Grenoble, France \\ 2 Sharif Univ. of Technology, Electrical Engineering Dept., Tehran, IRAN, \\ bahram.ehsandoust@gipsa-lab.grenoble-inp.fr, mbzadeh@sharif.edu, \\ christian.jutten@gipsa-lab.grenoble-inp.fr
}

\begin{abstract}
While Blind Source Separation (BSS) for linear mixtures has been well studied, the problem for nonlinear mixtures is still thought not to have a general solution. Each of the techniques proposed for solving BSS in nonlinear mixtures works mainly on specific models and cannot be generalized for many other realistic applications. Our approach in this paper is quite different and targets the general form of the problem. In this advance, we transform the nonlinear problem to a time-variant linear mixtures of the source derivatives.

The proposed algorithm is based on separating the derivatives of the sources by a modified novel technique that has been developed and specialized for the problem, which is followed by an integral operator for reconstructing the sources. Our simulations show that this method separates the nonlinearly mixed sources with outstanding performance; however, there are still a few more steps to be taken to get to a comprehensive solution which are mentioned in the discussion.
\end{abstract}

Keywords. Blind Source Separation, Nonlinear Mixtures, Independent Component Analysis

\section{Introduction}

Blind Source Separation (BSS) is the problem of extracting the source signals that have been mixed together in a number of observations without any information about the mixture model or the sources [7]. In the simplest form of the problem, the number of sources and the observations are the same, and the sources are assumed to be statistically independent. The problem is formulated as

$$
\mathbf{x}(t)=\mathbf{f}(\mathbf{s}(t)),
$$

where $\mathbf{x}(t)=\left[x_{1}(t), \ldots, x_{n}(t)\right]^{T}$ and $\mathbf{s}(t)=\left[s_{1}(t), \ldots, s_{n}(t)\right]^{T}$ are the observation and source vectors, respectively (and $n$ is the number of sources which is considered to be equal to the number of observations). The problem has been intensively studied since 1985 . The first idea for performing the separation was trying to make independent signals combining the observations; hence named 
Independent Component Analysis (ICA) [10],[6]. It leaded to many noticeable achievements when the mixing model is linear, and several algorithms have been proposed such as INFOMAX, [2], JADE [5], Normalized EASI. [4], SOBI. [3], and FastICA [16].

Many practical applications for BSS have been considered by now, which is still being diversified. Separating the signals in a Multiple Input Multiple Output (MIMO) situation, separating EEG signals from different parts of the brain, discovering the hidden parameters affecting economical indexes, discriminating different layers of the earth from the reflections of the emitted electromagnetic wave, etc. are a few samples of the applications of BSS in real world [12].

However, extending theses accomplishments to the nonlinear case is not straightforward. As indicated in [11], ICA (i.e. source independence) is not sufficient for separating sources which are nonlinearly mixed.

But there are many realistic applications for which the linear BSS model could not be applied; e.g. smart chemical sensor arrays [8], hyperspectral imaging [9], and removing show-through in scanned documents [17]. Therefore, studies on this issue were focused on specific applications with restricted mixing models. Post Nonlinear composition (PNL) [18], Convolutive Post Nonlinear mixture [1], Bi-Linear model [17], conformal mappings [15], and mappings that can be transformed to linear mixtures via a nonlinear function [13] are some of the objectives that have been attained in this regard. Moreover, the problem has been addressed in [14] using the velocity state-space of the observations for continuous-time sources more recently.

Our approach to this problem is quite distinct. In this work, we add a few assumptions that should be met in addition to the conventional ones which are discussed in the following section. However, the conditions are not restrictive in practical applications and, so, the approach is more general.

\section{The Main Idea}

Our approach in this paper is based on the assumption that if the mixed sources meet a few conditions, they can be blindly separated utilizing signal derivatives. The assumptions consist of:

1. The derivatives of the sources need to meet the separability conditions of the conventional BSS problem for linear mixtures. Especially, they are statistically independent.

2. The sources are supposed to be colored; they need to have temporal correlation.

3. The nonlinear mixing model is required to be an invertible mapping.

4. The nonlinear mixing model is time-invariant.

Apparently, these conditions are met when the sources are not originally identical. In other words, if the sources are mutually independent in terms of stochastic processes, their derivatives are mutually independent as well (which guarantees meeting the required conditions). 
The proposed method is based on the fact that the derivatives of the sources are mixed linearly even though the mixture model is nonlinear in general. Let $s_{1}(t)$ to $s_{n}(t)$ and $x_{1}(t)$ to $x_{n}(t)$ represent the source signals and the observations respectively. The above assumption can be written as

$$
\mathbf{x}(t)=\mathbf{f}(\mathbf{s}(t)) \Rightarrow \frac{\partial \mathbf{x}}{\partial t}=\mathbf{J}(\mathbf{s}(t)) \frac{\partial \mathbf{s}}{\partial t}
$$

where $\mathbf{f}$ and $\mathbf{J}$ correspond the nonlinear mixing function and its Jacobian matrix respectively. The key point here is that, as the above equation shows, although the signals are mixed nonlinearly, their derivatives are mixed linearly. Accordingly, the main steps of the separating approach are summarized below:

\section{Algorithm 1: Adaptive Algorithm for Time-Variant mixtures (AATV)}

1. Compute the derivatives of the observations;

2. Consider them as the inputs for a linear BSS algorithm for linear mixtures;

3. Separate the derivatives of the observations to get the derivatives of the sources;

4. The sources are supposed to be the integral of the results.

However, except the sensitivity of the proposed method to the noise due to the derivative computation which is not addressed in the current work, there are two main challenges that highly affect the performance of the mentioned algorithm. The first one is that the mixing matrix (Jacobian) is time-variant and changes over the time. Therefore the existing methods for BSS in linear mixtures, which usually assume that the mixing matrix is constant, must be modified before being used for this problem. It is worth noting that the alterations of the mixing matrix depend on both the nonlinear mixing model, and the dynamics of the sources. However if the nonlinear mixing function changes slowly with respect to the sources (at the state of saturation, as an extreme example), the variations of the sources may lead to relatively small changes in the mixing matrix.

The second issue is the cumulative error in the integral of the separated signals because of the slow convergence of the BSS procedure. It should be noted that because the mixing matrix is time variant, it is not possible to run a batch algorithm for separating the derivatives that would prevent the convergence error in the signals: an adaptive (and fast) algorithm is mandatory.

To overcome the first challenge, we use an adaptive and iterative algorithm, which tracks the separating matrix in each iteration. In this context, the traditional convergence never happens, since the mixing model may vary over the time even though the output signals become completely separated at a moment.

Utilizing an adaptive algorithm causes the slow convergence and hence the error accumulation (the second mentioned concern). As it is stated before, even converging to the exact separating matrix at a point does not mean that it will properly work for the remained samples of the signals; for the reason that the mixing matrix (Jacobian) may alter rapidly, which should be followed by the adaptive algorithm again. 
But the Jacobian matrix is not inherently time-variant. Actually, due to the assumption of mixing system to be time-invariant, its Jacobian is a timeinvariant function of the source as well. In fact, considering the linear BSS model resulted by derivative computation, it is seen a time-variant mixing matrix; because the Jacobian matrix is calculated at different points (of the varying sources). Thus, trying to find the nonlinear nature of the separating matrix in the BSS procedure, we face a time-invariant problem, which may be solved by a batch algorithm.

$$
\dot{\mathbf{x}}=\mathbf{J}(\mathbf{s}) \dot{\mathbf{s}} \longleftrightarrow \frac{\partial \mathbf{x}}{\partial t}=\mathbf{J}(\mathbf{s}(t)) \frac{\partial \mathbf{s}}{\partial t}
$$

In other words, we propose to estimate the nonlinear mixing model which exists behind the linear time-variant matrix. This idea is similar to the one used in batch algorithms for linear mixtures, where the estimated mixing model is adjusted after data convergence, and is then applied to the whole data. The only difference here is that the system is nonlinear and, so, nonlinear modeling is used for the function to be extracted.

As a consequence, the BSS procedure is split into two phases. At the first phase, an adaptive algorithm is run on the observation derivatives and the separated signals are constructed. Then we extract the nonlinear time-invariant model from the result. To do this, the data after convergence (of the algorithm) is used for nonlinear modeling of the mapping. The obtained model is then applied to the data as the second run to compensate the slow convergence error.

Finally, the separating algorithm for the blind separation of nonlinearly mixed sources can be summarized as follows:

\section{Algorithm 2: Adaptive Algorithm and Nonlinear Estimation (AANE)}

1. Compute the derivatives of the observations;

2. Consider them as the inputs for an adaptive and iterative linear BSS algorithm;

3. Estimate (e.g. by spline interpolation) the time-invariant nonlinear model (nonlinear modeling) of the time-variant linear separating system derived from the previous step (by utilizing only the data after convergence);

4. Apply the obtained model to the whole data (observations) again in order to separate the signals correctly and get the derivatives of the sources;

5 . The sources are the integral of the results.

\section{Simulation Results}

Simulating the proposed method, we have chosen a simple two-input two-output system with the integrals of a saw-tooth (named $s_{1}(t)$ ) and a sinusoid (named $\left.s_{2}(t)\right)$ signal as the input. The mixing model (4) is the same as the counter example [1], which was thought not to be separable at all.

$$
\left[\begin{array}{l}
x_{1} \\
x_{2}
\end{array}\right]=\left[\begin{array}{cc}
\cos \alpha-\sin \alpha \\
\sin \alpha & \cos \alpha
\end{array}\right]\left[\begin{array}{l}
s_{1} \\
s_{2}
\end{array}\right] \quad \text { where } \alpha=0.1 \times \sqrt{s_{1}^{2}+s_{2}^{2}} .
$$



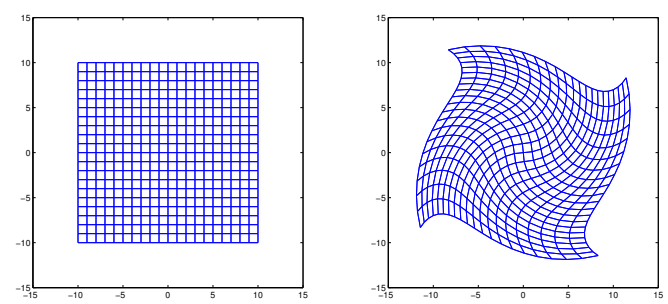

Fig. 1. Nonlinear mapping graph

Fig. 1 shows the nonlinear mapping of the mixing model (it can be shown that the model is invertible).

From the given model, calculating the Jacobian matrix is straightforward.

$$
J=\left[\begin{array}{ll}
\frac{\partial x_{1}}{\partial s_{1}} & \frac{\partial x_{1}}{\partial s_{2}} \\
\frac{\partial x_{2}}{\partial s_{1}} & \frac{\partial x_{2}}{\partial s_{2}}
\end{array}\right]=\left[\begin{array}{cc}
\cos \alpha & -\sin \alpha \\
\sin \alpha & \cos \alpha
\end{array}\right]\left[\begin{array}{cc}
1-s_{2} \frac{\partial \alpha}{\partial s_{1}} & -s_{2} \frac{\partial \alpha}{\partial s_{2}} \\
s_{1} \frac{\partial \alpha}{\partial s_{1}} & 1+s_{1} \frac{\partial \alpha}{\partial s_{2}}
\end{array}\right]
$$

In our simulation, Normalized-EASI [4] has been used as the linear BSS algorithm. This method is adaptive, iterative, and hence, suitable for the conditions of the problem. In addition, its equivariancy causes that its performance remains the same with respect to how much the sources are mixed together.

Performing the nonlinear modeling of the time-variant mixing model, we have used the down-sampled version (for increasing the speed of calculations) of the second half of the result (to make sure that we are using the outputs after convergence) after running Normalized-EASI on the data, to be modeled through a smoothing spline manner.

Obviously, it is not necessary to find the nonlinear mixing system for performing the proposed algorithm; it is sufficient to model the separating function instead. The models for the two outputs of the considered simulation are depicted in Fig. 2. In this figure, the blue circles are the converged samples of the elements of the separating matrix (which are resulted from the Normalized-EASI algorithm), and the plotted surfaces are the nonlinear model fitted to the data using smoothing spline. It should be also noted that we may directly model the nonlinear mapping of the two output signals as a function of the inputs (the derivatives of the observations) as well.

Finally, the obtained model is then applied to the whole data to adjust the result and is plotted for both sources in Fig. 3. This figure compares the original sources with the reconstructed ones by performing both the algorithm 1: AATV (signal named "AATV Result") and the algorithm 2: AANE (signal named "AANE Result"). In addition, table 1 shows the normalized mean squared error for both results of the both algorithms.

It is also shown in Fig. 3 that Normalized-EASI is a fast adaptive algorithm and overcomes the first challenge (the mixing matrix changes over the time) 

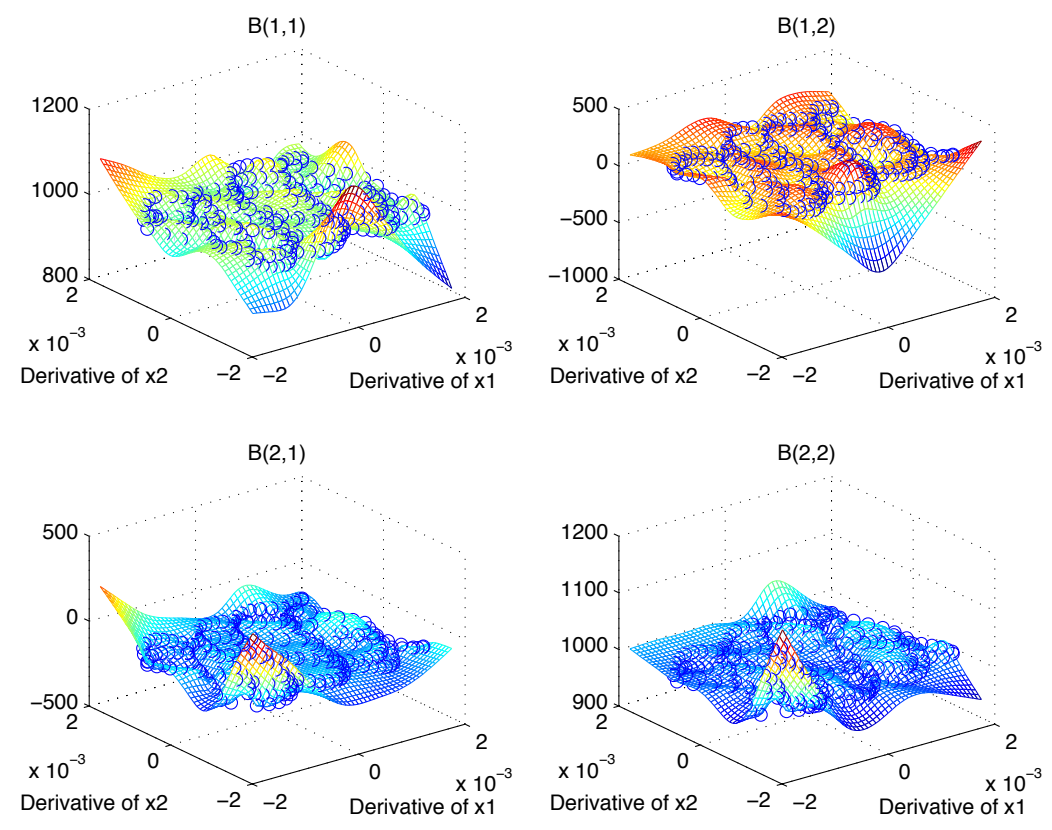

Fig. 2. Nonlinear modeling result of the elements of the separating matrix

Table 1. NMSE for AATV and AANE

\section{AATV AANE}

NMSE for the Source 11.60830 .0032

NMSE for the Source $20.4875 \quad 0.0244$

pointed out in the previous section. Nevertheless, the second issue (slow convergence and the cumulative error) dramatically damages the result if not amended by the nonlinear modeling.

\section{Discussion}

In this paper, a new method is proposed for BSS in nonlinear mixtures based on separating the derivatives of the signals. Our simulations show that the proposed algorithm works well and can be considered as a simple approach to a new class of techniques in this field.

This approach targets a more general class of practical BSS problems in nonlinear mixtures than the existing ones and is not limited to a specific mixing 

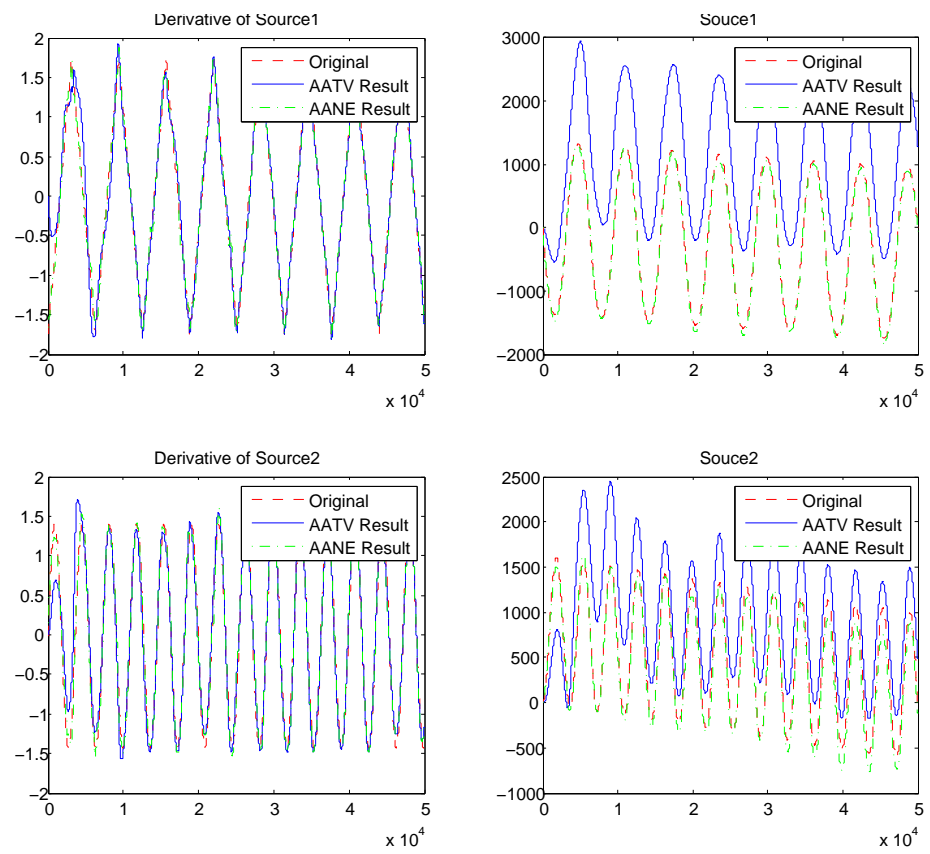

Fig. 3. Final extracted signals in comparison with the sources

model. However, there are a few concerns that should be thought about in this regard.

Firstly, the sources are supposed to be colored in the BSS problem (see the assumptions of the algorithm). This supposition is to make the derivatives suited for being separated by the adaptive BSS method for linear mixtures. It should be such that the adaptive algorithm can follow the variations. Solving the BSS for nonlinear mixtures through the proposed framework, we need that the BSS for the time-varying linear mixtures works properly.

Secondly, we have not considered the noise in the proposed algorithm. As we know, taking the derivatives amplifies the noise, and hence, makes the method more sensitive to the noise. At last, we should note that there exist the traditional ambiguities of BSS in linear mixtures (scaling and permutation) in the proposed method as well. However, these ambiguities do not differ for different samples of the signals (because in the proposed method, the unique extracted nonlinear model is applied on the whole data).

\section{Acknowledgement}

This work is partly funded by the European project 2012-ERC-Adv-320684 CHESS. 


\section{References}

1. Babaie-Zadeh, M.: On blind source separation in convolutive and nonlinear mixtures. Ph.D. thesis, INP Grenoble (2002)

2. Bell, T., Sejnowski, T.: An information-maximization approach to blind separation and blind deconvolution. Neural Comutation 7(6), 1004-1034 (1995)

3. Belouchrani, A., Abed-Meraim, K., Cardoso, J., Moulines, E.: A blind source separation technique using second-order statistics. IEEE transaction on Signal Processing 45(2), 434-444 (February 1997)

4. Cardoso, J.F., Laheld, B.: Equivariant adaptive source separation. IEEE Trans. on SP 44(12), 3017-3030 (December 1996)

5. Cardoso, J.F., Souloumiac, A.: An efficient technique for blind separation of complex sources. In: Proc. IEEE Signal Processing Workshop on Higher-Order Statistics. pp. 275-279. South Lac Tahoe, USA (CA) (June 1993)

6. Comon, P.: Independent component analysis, a new concept? Signal Processing 36(3), 287-314 (1994)

7. Comon, P., Jutten, C.: Handbook of Blind Source Separation: Independent component analysis and applications. Academic press (2010)

8. Duarte, Tomazeli, L., Jutten, C.: Design of smart ion-selective electrode arrays based on source separation through nonlinear independent component analysis. Oil \& Gas Science and Technology-Revue dIFP Energies nouvelles 69(2), 293-306 (2014)

9. Golbabaee, M., Arberet, S., Vandergheynst, P.: Compressive source separation: Theory and methods for hyperspectral imaging. Image Processing, IEEE Transactions on 22(12), 5096-5110 (2013)

10. Hérault, J., Jutten, C.: Space or time adaptive signal processing by neural networks models. In: Intern. Conf. on Neural Networks for Computing. pp. 206-211. Snowbird (Utah, USA) (1986)

11. Hosseini, S., Jutten, C.: On the separability of nonlinear mixtures of temporally correlated sources. IEEE signal processing letters 10(2), 43-46 (February 2003)

12. Hyvärinen, A., A., Karhunen, J., Oja, E.: Independent component analysis, vol. 46. John Wiley \& Sons (2004)

13. Kagan, A.M., Linnik, Y.V., Rao, C.R.: Extension of darmois-skitovich theorem to functions of random variables satisfying an addition theorem. Communications in statistics 1(5), 471-474 (1973)

14. Levin, D.N.: Performing nonlinear blind source separation with signal invariants. Signal Processing, IEEE Transactions on 58(4), 2131-2140 (2010)

15. Hyvärinen, A., Pajunen, P.: Nonlinear independent component analysis: Existence and uniqueness results. Neural Networks 12, 429-439 (1999)

16. Hyvärinen, A.: Fast and robust fixed-point algorithms for independent component analysis. IEEE Transactions on Neural Networks 10(3), 626-634 (1999)

17. Merrikh-Bayat, F., Babaie-Zadeh, M., Jutten, C.: Linear-quadratic blind source separating structure for removing show-through in scanned documents. International Journal on Document Analysis and Recognition (IJDAR) 14(4), 319-333 (2011)

18. Taleb, A., Jutten, C.: Source separation in post nonlinear mixtures. IEEE Transactions on Signal Processing 47(10), 2807-2820 (1999) 